\title{
EL ROL DE LA ESCUELA EN LA \\ CONSTRUCCIÓN DEL DESARROLLO \\ RURAL: \\ EXPERIENCIAS ARGENTINAS
}

Natalia Millán'

\section{Resumen}

La globalización, los procesos de desterritorialización de la producción, el surgimiento del desarrollo local y la instalación de la sociedad del conocimiento, inclusive en los ámbitos rurales, implican un serio cuestionamiento para la educación rural, que debe enfrentarse a un proceso de modemización y reconstrucción para no quedar excluida de los procesos sociales actuales.

Se analizan en la presente ponencia algunos itemes de la política educativa para el sector rural en Argentina, y se reflexiona, a partir de dichas experiencias, sobre cómo seguir de cara al futuro.

Palabras claves: educación rural, descentralización educativa, desarrollo local, participación.

\section{Abstract}

Social processes such as globalization, non territorial productive processes, sprouting of local development and the arising of knowledge society -even in the rural context- bring serious implications for rural education, which has to face a process of modernization and reconstruction in order to avoid the exclusion of current social processes.

Different aspects of educational politics in Argentinean rural sectors are analyzed in this article, and a reflection is made about this analysis in order to determine the path to follow in the future.

Keywords: rural education, decentralize education, local development, participation.

1 Profesora pasante, noviembre 2006, Sociöloga Rural en el Plan de Desarrollo Rural del Programa de Participación Local y Desarrollo Humano del Instituto de Desarrollo Rural, Mendoza, Argentina.

Recibido: 30 de octubre-2006 - Aprobado: 15 de noviembre-2006 


\section{Introducción}

Ce observa que hoy, la demanda del campo a la ciudad es distinta a la de hace cuarenta años. La ruralidad, como fue definida en términos tradicionales, ya no existe más. La industrialización de la agricultura y la urbanización de las comunidades rurales acabó con la ruralidad tradicional, pero no con lo rural.

Lo rural comprende un tipo particular de relaciones sociales con un componente personal predominante en territorios con baja densidad de población. Según Gómez (2002), "Esta relación personal tiene una fuerte base en las relaciones vecinales, con relaciones de parentesco prolongadas entre los habitantes" (p. 33).

La imagen de la comunidad campesina autosuficiente ha desaparecido y ha sido sustituida por la mercantilización y monetarización de la economía. Los campesinos deben buscar una serie de bienes y servicios que no están disponibles en la explotación. Estas vinculaciones de la producción campesina con la sociedad mayor se denominan articulaciones. Una de las principales vías de articulación de los campesinos es mediante los mercados.

En este marco de análisis, se hace un estudio de tipo descriptivo de la evolución de los modelos educativos a través del tiempo en Argentina. Posteriormente, se analizan algunas conceptualizaciones básicas respecto a la educación, la escuela, el desarrollo local y el territorio en relación con el rol de la educación rural en Argentina.

La ponencia finaliza con la presentación de políticas concretas que involucran a la educación y a la escuela rural de una u otra forma, en los distintos niveles del Estado: Nacional, Provincial y Municipal. Asimismo, se destacan algunas conclusiones respecto a lo positivo y lo negativo de las experiencias presentadas, de cara al futuro.

\section{La evolución del sistema educativo argentino}

Se debe considerar la educación, como una práctica social o un conjunto de prácticas sociales, consistente en socializar mediante la enseñanza de conocimientos (Cullen, 1997). Partiendo de esta conceptualización, se ha elaborado una periodización histórica de los modelos educativos en Argentina, estableciendo relaciones en el interior de cada modelo entre política y educación. Por tal motivo, se describen los modelos políticos desde la segunda mitad del siglo XIX en adelante, tal y como se dieron en este país. 
Modelo oligárquico. Durante la segunda parte del siglo XIX y las primeras tres décadas del siglo XX, existió un pacto de dominación vigente en América Latina, que podría caracterizarse como un Estado oligárquico. Este Estado consolidó las naciones y generó una estabilidad política relativa, basando su modelo político en la exportación masiva de productos agropecuarios, con una política económica antiindustrialista (Torres y González Rivera, 1994).

Como parte de los estados oligárquicos, se desarrolló en Argentina el sistema de educación pública, buscando establecer los fundamentos de la nación y la ciudadanía. El papel y la función atribuida a la educación pública eran la creación de un sujeto pedagógico disciplinado. En este contexto, la misión, la ideología y entrenamiento del magisterio normalista fueron marcados a fuego por la filosofia liberal y positivista (Puiggrós, 1990).

En este marco, es promulgada por Julio A. Roca el 8 de julio de 1884, la Ley $N^{\circ} 1420$, quien legisló para la Capital Federal y los Territorios Nacionales; la ley hacía referencia a la escuela primaria. Se planteó como síntesis de un modelo educativo que obedecía a un modelo político, el cual promovía la inmigración, y a su vez se sustentaba en un modelo económico agroexportador.

La misión de esta ley era educar al soberano, teniendo en cuenta que llegaban inmigrantes españoles e italianos analfabetos a Argentina. Es en este sentido, que se puede captar la importancia de la obligatoriedad y gratuidad de la escuela primaria, que a través del desarrollo moral, intelectual y físico de todo niño entre 6 y 14 años, llevaba a la formación de ciudadanos. La Ley $\mathrm{N}^{\circ} 1420$ buscaba la unidad nacional, la homogeneización y alfabetización del país que estaba surgiendo. Se planteaba universalizar la enseñanza primaria a toda la población.

En 1905, se sancionó la Ley Láinez (Ley N²874). Fue pensada como instrumento de colaboración entre la Nación y las provincias. Su objetivo era crear escuelas en las provincias, en zonas rurales donde no hubiera educación primaria; pero se distorsionaron sus fines, porque las escuelas nacionales fueron establecidas en lugares donde ya había escuelas, y se creó competencia entre las escuelas nacionales y las provinciales.

Estado interventor. Con el desmoronamiento del pacto oligárquico, a partir de los años 30, se abrió una nueva época política en América Latina marcada por trazos de un nuevo modelo de desarrollo y nuevos modelos de Estado. En Argentina comenzó a gestarse lo que luego se conocería como 
peronismo. Si bien sería ficticio tratar de encontrar una versión latinoamericana del Estado de Bienestar Social, se puede decir que las políticas de bienestar social llevadas a cabo por el populismo (que se representó en el peronismo en Argentina) tuvieron un sinnúmero de paralelos con el Estado de Bienestar Social (Torres y González Rivera, 1994).

En lo que a educación respecta, se puso énfasis en que los hijos de la clase obrera y la población en general, pudieran acceder a la educación pública, gratuita y laica. También se trató de concientizar a la ciudadanía y, especialmente a la clase obrera, de cuáles eran sus derechos como ciudadanos y como trabajadores.

Crisis y ajuste estructural. Si bien las cifras de la expansión educativa entre 1960-1970 en América Latina dan cuenta de las tasas más altas de crecimiento educacional en el mundo, con índices de crecimiento para la educación primaria de $167,5 \%$, para la educación secundaria de $247,9 \%$ y de $258,3 \%$ para la educación superior (Torres y González Rivera, 1994); a pesar de ello, durante los setenta y los ochenta, tuvo lugar un nuevo proceso de reorganización de la división internacional del trabajo y del capital, que afectó a la educación.

Este proceso de reorganización mundial se basó en la adopción de estrategias globales de producción, implementadas por las compañías multinacionales, que crearon un mundo económico y políticamente más interdependiente, con fuertes presiones para recortar el valor de la fuerza de trabajo, reemplazando las fórmulas económicas keynesianas por nuevas ortodoxias económicas, con ideologías de mercado.

A la luz de esta situación mundial, las nuevas democracias latinoamericanas, con su enorme deuda externa, sus limitaciones de acumulación doméstica de capital y la crisis fiscal del Estado, para restaurar el desarrollo económico, adoptaron los préstamos-puente del FMI y los préstamos de ajuste estructural del Banco Mundial (BM).

En relación con la educación, el ajuste estructural y los préstamos otorgados por el FMI y el BM impusieron un número de condiciones: nuevos esfuerzos orientados a transferir el costo de los servicios a los usuarios, incrementar la participación del sector privado en educación, privatización, reducir el costo de la educación afectando el nivel de salarios, y promover la descentralización de los servicios educativos como una medida de redefinir las relaciones de poder y educativas entre los gobiernos nacionales (federales), provinciales y municipales (Landi, 2002). 
Este proceso de ajuste estructural fue acompañado en Argentina por un Estado ausente en lo social y por una sucesión de dictaduras militares que derrocaron a los gobiemos constitucionales. Bajo los regímenes militares, el sistema educativo promovió un modelo de ciudadano pasivo, que no tenía que opinar sobre política, ni reclamar por sus derechos ciudadanos. Simultáneamente desde las juntas militares, que reemplazaban al Estado, se planteaba que toda manifestación o reclamo de los derechos legítimos sería considerada como una forma de subversión (Torres y González Rivera, 1994).

En 1978 es sancionada la Ley $N^{\circ} 21809$. A través de esta ley se transfirieron las escuelas primarias nacionales a las provincias. Poco a poco, la nación dejó de pasar fondos a las provincias para educación. Se transfirieron a las provincias las escuelas, pero no se transfirieron recursos ni financiamiento.

Década de los noventa. En la década de los ochenta, retornó la democracia en Argentina, junto con un proceso de reconceptualización de la vida social, democrática y política. Con el inicio de la década de los noventa, la educación y el conocimiento se colocaron nuevamente en un lugar central en el debate acerca de las estrategias de desarrollo económico y social de los países latinoamericanos. La recuperación de una perspectiva optimista acerca del aporte de la educación a la sociedad estuvo sustentada en la necesidad de retomar la senda del conocimiento y de mejorar los niveles de equidad, partiendo de la crisis económica y la profundización de la pobreza ${ }^{2}$.

Se ubicó así la creación, incorporación y distribución del conocimiento como el factor principal para las tareas de conocimiento y equidad social que se habían colocado como prioritarias para el desarrollo de América Latina.

Comenzaron a tener una fuerte presencia en la región nuevas perspectivas acerca de la importancia estratégica de la educación, que ya se estaban convirtiendo en hegemónicas en los países centrales.

En relación con este nuevo impulso a la educación mundial, en Argentina se sancionó en 1993 la Ley № 24195, conocida como la Ley Federal de Educación. Esta ley planteaba un nuevo sistema educativo que implicó la descentralización de la educación, una reestructuración del sistema, una renovación en los contenidos, mayor capacitación y el Estado prometió más fondos para la educación. En cuanto al modelo de ciudadanía que implica la educación, se comenzó a educar a los ciudadanos como consumidores de bienes y servicios que se ofrecen en el mercado.

2 El nucvo enfoque con que se abordó la problemática cducativa quedó delineado en el dosumento "Educación y conocimiento, eje de la transformación productiva con equidad", publicado por la CEPAL-UNESCO en 1992. 
Esta evolución histórica, que relaciona los modelos políticos y educativos, permite afirmar que el contexto político y económico en que se dicta la Ley Federal de Educación está impregnado por las ideas neoliberales, según las cuales los ciudadanos se reducen sólo a ser productores y consumidores de bienes materiales.

La Ley Federal de Educación (Ley $N^{\circ}$ 24195), sancionada en abril de 1993, es la que se encuentra vigente en la actualidad. Es la primera ley general de educación, porque abarca todos los niveles del sistema educativo: Educación Inicial, Educación General Básica, Polimodal, Superior. Los hacedores de esta propuesta plantean que ella implica cambios sustantivos y profundos en el sistema educativo nacional para elevar la calidad de la educación y para alcanzar mayores niveles de justicia social y de equidad en la distribución de saberes y conocimientos (Ministerio de Cultura y Educación de la Nación, 1994).

Se implementan diez años de escolaridad obligatoria a través de la Educación General Básica (EGB), los cuales comprenden dos niveles educativos: la Educación Inicial y la EGB. La Ley Federal de Educación también prevé el Polimodal (en reemplazo de la antigua escuela secundaria), la Educación Superior, la Educación Cuaternaria y una serie de Regímenes Especiales.

La propuesta de esta ley es elaborada con base en el modelo de legislación educativa española, el cual ya no se aplicaba en España por considerar que no había dado resultados positivos.

Hace dos meses, fue promulgada la Ley de Educación Técnico Profesional, que regula y ordena el nivel medio y superior no universitario del sistema educativo nacional y la formación profesional. Es de aplicación nacional, pero respeta los criterios provinciales y las diversidades regionales, articulando la formación general y profesional. Esta ley aún no se ha implementado.

Tras observar el gran número de estudiantes egresados del Polimodal que fracasan en su intento por ingresar en las universidades argentinas y los bajos niveles de calidad educativa, y a la luz de las falencias en la implementación de la Ley de Educación Federal, el gobierno nacional ha decidido regresar a la vieja Ley $\mathrm{N}^{\circ} 1420$, pero incorporando modificaciones; para lo cual durante los meses de junio, julio y agosto del 2006, se les entregó a los maestros de todo el país el proyecto con las modificaciones correspondientes para que lo analizaran, corrigieran e incorporaran aquello que consideren pertinente. A la fecha, se anuncia una consulta similar a la sociedad, pero por el momento sigue vigente la Ley de Educación Federal. 
El Banco Internacional de Reconstrucción y Fomento ha resuelto otorgar un financiamiento a la República Argentina por un monto de ciento cincuenta millones de dólares estadounidenses (US\$ 150.000.000) en el marco del Convenio de Préstamo BIRF N ${ }^{\circ} 7353 \mathrm{AR}$, comprometiéndose dicho Banco a asistir financieramente a la República Argentina, con el fin de posibilitar la ejecución del "Proyecto de Mejoramiento de la Educación Rural", que se desarrollará bajo la supervisión general del Ministerio de Educación, Ciencia y Tecnología. Dicho préstamo fue firmado por el Presidente de la Nación, en octubre del año 2006.

\section{Desarrollo, territorio y conocimiento en relación con el rol de la educa- ción rural en la actual sociedad globalizada}

En la utilización del término desarrollo como concepto vinculado a las ciencias sociales, muchos autores encuentran el origen de su utilización en las ciencias sociales en el discurso pronunciado por el Presidente de los Estado Unidos Truman con motivo de asumir la presidencia en enero de 1949. En dicho discurso, tras enumerar otras tres temáticas, incluyó un cuarto punto en el que comentaba: “...Debemos lanzarnos a un nuevo y audaz programa que permita poner nuestros avances cientificos y nuestros progresos industriales a disposición de las regiones insuficientemente desarrolladas para su mejoramiento y crecimiento económico".

Este discurso es tomado por muchos antropólogos como el origen del término desarrollo con una acepción vinculada a lo económico y social en relación con un tercer mundo que debía ser salvado de las influencias comunistas de la URSS y luego de la experiencia cubana en el continente.

El término desarrollo guarda una estrecha relación con el evolucionismo de tipo darwinista, al considerar que hay estadios de evolución inequívocos en las sociedades, y que es responsabilidad de las sociedades occidentales más avanzadas hacerles conocer dicho desarrollo a los países más atrasados y empobrecidos.

En el caso específico de la educación rural y de la generación de desarrollo en comunidades rurales, se debe ser muy cuidadoso respecto a qué significan dichos términos, ya que construir procesos de desarrollo según los parámetros urbanos en zonas rurales, puede generar un rechazo de la población a participar. Se parte aquí de considerar que el desarrollo de las comunidades rurales ha de originarse de acuerdo con las demandas concretas de la población en la que se trabaja y no a partir de planes de desarrollo 
de organismos internacionales y nacionales elaborados detrás de escritorios, sin relación real con lo que pasa en las zonas rurales en general y en cada comunidad rural en particular. No hay desarrollo posible, si no es aquel que respete los valores culturales y estilos de vida de la población con la cual se generan y construyen.

Al término desarrollo se le suele asociar en la actualidad a procesos locales. Cuando hablamos de local, es importante reconocer que, aun en el contexto de la globalización, el mundo sigue siendo terriblemente heterogéneo, y es fundamental entender la existencia y generación de dinámicas locales en el marco de sistemas productivos localizados geográficamente y la emergencia de formas espaciales de acción colectiva. En ella se encuentran contenidas redes localizadas entre agentes, que se usan para comprender el funcionamiento local de los productores. El funcionamiento en red permite salir del aislamiento, facilita la transmisión de información y aprendizaje, y define colectivamente las normas y las reglas comunes concernientes a la propiedad de los productos e intercambio de saberes.

Los organismos de crédito internacional (Banco Mundial, BID, etc.) también han modificado su visión de desarrollo con una visión más amplia e integral, respecto a la que se desprendía del Consenso de Washington. Hasta la CEPAL, que impulsó el desarrollismo en la región a partir de los 50, una autocrítica a su visión unilateral y cuantitativa de desarrollo. Hoy plantean que las principales líneas de acción son la educación y el conocimiento como bases de la transformación productiva con equidad, la cooperación intrarregional, la variable ambiental y la articulación entre desarrollo y gobernabilidad (Di Pietro, 2003).

En el marco de este paradigma, el rol de la escuela permite que algunas comunidades adquieran la capacidad específica para generar beneficios para sus pobladores, en relación con conocimientos aprendidos, dando cuenta de que las comunidades, sus instituciones y logros no son ahistóricos, eternos e inamovibles, sino que evolucionan en el tiempo y el espacio y son resultado de una acumulación de saberes, esfuerzos y mucho trabajo realizado a través del tiempo (Gilly y Torre, 2000).

En el marco del desarrollo local el término territorio es reconceptualizado; según Arocena (1995), "es un territorio con determinados límites, portador de una identidad colectiva, expresada en valores y normas interiorizadas por sus miembros y que conforma un sistema de relaciones de poder constituidas en torno a procesos locales de generación de riquezas... una 
sociedad local es un sistema de acción sobre un territorio limitado capaz de producir valores comunes y bienes localmente gestionados" (p. 38).

La utilización de este concepto favorece la construcción del territorio, su población, sus valores y creencias, sus instituciones, sus redes de relaciones y de poder como un todo en constante transformación, permitiendo ver el territorio como algo más que un mero espacio. En lo local, el espacio se convierte en territorio real, vivo, interpelante, que obliga a situarse en una perspectiva integral, el entomo así se convierte en activo, dinámico, facilitador de cambio tecnológico, estimulador de la creatividad. Hablar de desarrollo endógeno significa hacer referencia a la existencia de un proceso emprendedor e innovador, en el que el territorio no es un receptor pasivo de las intervenciones externas, sino que tiene una estrategia propia que le permite incidir en la dinámica general.

En ese marco, se debe comprender el significado de la identidad local, que implica la pertenencia a una comunidad y la posibilidad para los individuos de reconocerse en una identidad colectiva compartida; así, la relación del sujeto con su territorio es lo que determina la identidad. Finalmente, la identidad propia de cada territorio es un sustento para su desarrollo productivo.

En la actualidad, se vive una declinación del Estado-Nación como recurso identificatorio; asimismo, se acelera la globalización, y junto con el aumento de las identidades globales, se produce un auge de la reivindicación de las identidades locales, lo local afirma las diferencias.

Es importante señalar el papel que tiene la participación social en los procesos de desarrollo; esto implica que los actores participen en todo el proceso de intervención social, desde el diagnóstico hasta la evaluación, capitalizando así su conocimiento de la realidad local. Esto requiere comunidades fuertes, dinámicas, activas.

Con el surgimiento de las identidades locales, los municipios adquieren nuevos roles, entre los que destaca convertirse en agentes de desarrollo, que, como tales, siguiendo a Coraggio, tienen que atender tres grandes desafios: competitividad, gobernabilidad y un desarrollo humano sustentable. Sin embargo, uno de los principales problemas que enfrenta es la autonomía, que abarca varias esferas: financiera, institucional y administrativa, para la cual no se encuentran capacitados. De esta manera, la noción de desarrollo no sólo queda en lo económico, se entiende este como un hecho más complejo que engloba una pluralidad de dimensiones y no puede ser sólo medido desde lo cuantitativo.

En este escenario, la escuela puede ser visualizada como un agente de desarrollo privilegiado, con capacidad de generar empoderamiento en la 
comunidad, procesos de desarrollo participativo y círculos virtuosos de innovación y conocimiento que permitan el desarrollo armónico de la comunidad.

La educación es un proceso que dura toda la vida, es decir, no hace referencia únicamente al paso por la escuela; también es educación la socialización primaria y toda enseñanza no formal que los individuos reciben a lo largo de su existencia. La educación, en su sentido amplio, es una práctica social o un conjunto de prácticas sociales, consistente en socializar mediante la enseñanza de conocimientos (Cullen, 1996).

Complementariamente, la educación es un proceso constitutivo de la experiencia humana, y la escolarización es uno de los recortes de ese proceso educativo más amplio. Tanto en los procesos educativos más generales como en la educación escolar, realizamos aprendizajes de naturaleza diversa y construimos diferentes representaciones y valores (Gentili, 2000).

Por otra parte, la escuela tiene una función pública, enseñar, que es algo distinto de ser meramente un aparato reproductor del Estado, o un mero movimiento social reivindicativo de intereses diferenciados. La escuela posee una función esencial que la define por ser la institución, solidaria y autónoma, de enseñanza de contenidos educativos, legitimados públicamente (Cullen, 1996). También es un sitio caracterizado por una pluralidad de lenguajes conflictivos y luchas, donde los maestros, los alumnos, los padres y los administradores escolares con frecuencia tienen opiniones diferentes en cuanto a la forma en que se deben definir y entender las experiencias y prácticas de la escuela (Giroux, 1993).

Tal como plantea Roberto Vidal, Secretario y Director de Educación de la Sociedad Rural Argentina, los grandes cambios tecnológicos de los últimos años han abierto para el sector agroindustrial argentino un sinnúmero de oportunidades que, a su vez, ponen en evidencia carencias educativas. Este déficit se advierte también en la educación rural que no está de acuerdo con los niveles del conocimiento alcanzado en el siglo XXI. Las consecuencias se hacen visibles en una paradoja: por una parte, la falta de empleo; por la otra, las empresas se quejan, porque tienen dificultades para obtener mano de obra calificada.

Este es el contexto en el que se inserta la educación rural a principios del siglo XXI, con fracasos, desafios y retos sobre los cuales reflexionar para seguir adelante. Según se resuelva este acertijo, se irá perfilando el panorama de la educación rural de cara al futuro. Para ello, es importante recordar y aprender de la historia latinoamericana, para no cometer los mismos equívocos y rescatar lo positivo de las experiencias previas, ya que los pueblos 
sin memoria histórica carecen no sólo de pasado, sino también de presente y futuro, porque están condenados a repetir eternamente los mismos errores.

\section{El rol del Estado en la educación rural visualizado en tres casos a distin- tos niveles: nacional, provincial y municipal}

\section{Estado Nacional}

En este contexto de cambios en las políticas educativas, el Ministerio de Educación, Ciencia y Tecnología de la Nación ha definido entre las prioridades de política educativa para la Gestión 2004-2007, la atención a las diferentes temáticas que se relacionan con la educación rural ${ }^{3}$.

Las escuelas rurales tienen una alta relevancia cuantitativa en Argentina, siendo 11.324 unidades educativas de EGB 1-2 (sobre un total de 22.991), y de EGB 35.693 (sobre un total de 16.021).

Las escuelas de zonas se constituyen en un lugar de referencia para las comunidades a las que pertenecen, deben dar respuesta a las necesidades educativas de las poblaciones aisladas considerando el contexto particular de cada situación y promoviendo el enriquecimiento del capital cultural de sus estudiantes. Por otra parte, por las condiciones de vida de estas comunidades, en un alto porcentaje, los niños y jóvenes que concurren a las escuelas rurales pertenecen a los sectores más pobres de esos territorios.

Los indicadores educativos de estas escuelas dan cuenta de situaciones específicas. Es común que los niños y las niñas ingresen tardíamente al sistema, entre otros aspectos, por la escasa oferta educativa para el Nivel Inicial y por las condiciones de vida particulares de las familias; también sucede que, en el transcurso del año lectivo (generalmente durante marzo), un cierto número de niños y jóvenes se ausenta por períodos prolongados, debido a la incorporación a trabajos temporarios junto a sus familias, tales como la cosecha de vid y frutales, o debido a tener que cuidar de sus hermanos menores en ausencia de sus padres.

En muchos casos, los bajos índices de rendimiento se vinculan a las inadecuadas propuestas educativas, incidiendo en las repitencias reiteradas, gencrando altas tasas de sobreedad y serias dificultades para mantener la escolaridad.

El Ministerio propone considerar las particularidades de la educación rural en la definición de las políticas educativas nacionales y provinciales que

3 Dicha propuesta se describe con mayor detenimiento en el documento titulado: Educación. Plan de Acción 2005-2007, claborado por el Ministerio de Educación, Ciencia y Tecnologia, Presidencia de la Nación. 
requieren para su implementación; previendo los modos de articulación entre ámbitos rurales y urbanos, delimitando el lugar del Estado y de las ONG, profundizando la vinculación entre lo educativo y productivo, analizando las implicaciones del desarrollo local en el marco de lo global.

La atención a la educación rural demanda la acción articulada de la Nación y las provincias en la definición de propuestas que respondan a lineamientos generales; pero a la vez exige toma de decisiones situadas desde las particularidades de cada zona e institución. También considera dicho organismo estatal, trabajar con el plurigrado o sección múltiple, como eje del trabajo en los diversos ciclos y niveles del Sistema Educativo y en las propuestas de formación docente inicial y continua.

Para llevar adelante dichas propuestas, el Ministerio propone la designación en cada jurisdicción de un referente que articule la tarea entre el equipo de ruralidad de la Dirección Nacional de Gestión Curricular y Formación Docente y los diferentes equipos de los ministerios provinciales, así como la incorporación en la formación de base, la perspectiva de la ruralidad, tanto en las asignaturas como en las instancias de práctica y residencia, fomentando posibles postítulos de enseñanza en ruralidad.

Entre otras se destacan propuestas de proyectos escolares de desarrollo local vinculados con la perspectiva del desarrollo local de las comunidades en las cuales están insertas las escuelas. Trabajo que compartirán el Instituto Nacional de Tecnología Agropecuaria (INTA) y el Programa Social Agropecuario (PSA), mediante un futuro convenio con la Secretaría de Agricultura. Otra propuesta que vale destacar es la creación de una EGB rural, para ser aplicada desde el 2006 en adelante. Se propone que todo alumno que asista a escuelas rurales complete su escolaridad básica en el sitio donde cursó los dos primeros ciclos de la EGB.

Cabe destacar que estas propuestas del gobiemo nacional se encuentran elaboradas, pero no han sido aún implementadas de hecho en las escuelas rurales del país.

\section{El Estado provincial y los organismos mixtos: el caso del Plan de Desa- rrollo Rural del Instituto de Desarrollo Rural (IDR)}

El Plan de Desarrollo Rural, a través del Programa de Desarrollo Humano, lo ejecutan promotores de desarrollo que trabajan en el territorio con la comunidad y desde las escuelas rurales. En este caso, el eje está puesto en la escuela rural como espacio de nexo con la comunidad, ya que a través 
de los niños y las niñas en edad escolar, se puede trabajar con sus padres y madres para ver las dificultades sociales y productivas que atraviesan como comunidad.

El plan cuenta con un grupo coordinador para toda la provincia y con supervisores en cada uno de los departamentos; ahí se trabaja con un grupo de promotores, que están en contacto con la población. A través de la elaboración de los diagnósticos, la comunidad debe asumir sus problemas y buscar la forma de solucionarlos; el plan busca que las personas comiencen a organizarse para la autogestión en aras de solucionar sus problemas y necesidades.

En este momento, se está trabajando con 120 comunidades en la provincia de Mendoza. Se busca establecer vínculos con la gente; a partir de estas intervenciones han surgido en algunos poblados organizaciones sociales, con el fin de lograr la consolidación de grupos dentro de las localidades rurales.

Respecto a la elaboración de proyectos, se cuenta, en este momento, con 98 proyectos productivos elaborados, respecto a 408 problemáticas sociales. Estos proyectos se están implementando a través del plan "Manos a la obra”. En cuanto a la vinculación con otras instituciones, se está trabajando en conjunto con la Dirección General de Escuelas (DGE), en temas vinculados a capacitación, tanto formal como informal.

La definición de ruralidad con la que se mueve el plan es elaborada por la gente, por lo cual se observa que parte de una definición de territorio vinculada a la población.

El marco teórico y metodológico en el que se inserta el plan se ha trabajado desde el enfoque elaborado por el Grupo de Investigaciones Agrarias de la Universidad Cristiana de Chile, con autores como Sergio Ríos y Miguel Bahamondes. Los aspectos de manejo de herramientas participativas para el diagnóstico han sido aportados por material de la GTZ $^{4}$ y el IICA ${ }^{5}$; y en cuanto a la elaboración de proyectos se ha utilizado bibliografia de la $\mathrm{FAO}^{6}$.

Se trabaja con técnicas participativas de carácter cualitativo para la elaboración de diagnósticos y proyectos. Hasta el momento, no se ha elaborado ningún tipo de indicadores para medir el campo, estos están en proceso de elaboración. Las bases de datos hechas contienen información de los diagnósticos participativos y algunas estadísticas del trabajo con la comunidad.

\footnotetext{
4 Sigla correspondiente a la Agencia de Cooperación Alemana.

5 Sigla del Instituto Interamericano de Cooperación para la Agricultura.

6 Sigla correspondiente a la Food and Agriculture Organization (Organización para la Alimentación y la Agricultura).
} 


\section{El municipio: educación de la población rural en el departamento de Las} Heras, un ejemplo de municipio ausente

Desde el municipio de Las Heras, se solicitó se midieran las condiciones de vida y productivas de los distritos rurales del municipio; para ello se tomó como unidad de análisis la población rural del departamento, dividiéndola por distritos, no las propiedades que habitan, ni la producción agrícola ni a lo que se dedican, sino los pobladores de la zona, quienes habitualmente viven y desarrollan sus labores en las zonas rurales; la prueba piloto del relevamiento se realizó en el distrito El Pastal.

Para iniciar la investigación, se trabajó con informantes claves, que comentaron su visión sobre la situación del distrito y colaboraron en la definición espacial del área objeto de la presente investigación; si bien los distritos tienen límites político-administrativos, los límites de influencia de la localidad en la realidad son distintos. Simultáneamente, se ubicaron los centros de salud y las escuelas en un mapa de cada distrito. Todos estos aspectos de aproximación al campo permitieron explorar la situación de cada distrito, contribuyendo a la elaboración del instrumento de medición y sus correcciones.

La segunda etapa metodológica fue la recolección de datos. Para ello, se diseñó un instrumento de medición adecuado, un cuestionario, para realizar entrevistas por encuesta a los productores, que permitió indagar aspectos socioeconómicos y productivos de los habitantes del distrito El Pastal.

Se trabajó con la Delegada Municipal del distrito y se realizó el relevamiento a beneficiarios del Plan Jefes y Jefas de Hogar ${ }^{7}$ residentes en el distrito, quienes fueron capacitados para desempeñarse como encuestadores, además, aportaron comentarios para realizar mejoras en el cuestionario en función de conocer la realidad de cada uno de los distritos. Se entrevistaron 147 productores de la zona. Se trabajó con la metodología cuantitativa en un principio, a través del armado de bases de datos (en formato SPSS).

El departamento de Las Heras se encuentra ubicado al norte de la provincia de Mendoza y sus límites son: al norte San Juan; al oeste la Rep. de Chile; al este los departamentos de Lavalle, Guaymallén, Godoy Cruz y Capital; al sur el departamento de Luján de Cuyo y Capital. La Cordillera de los Andes margina la parte oeste y sus pasos conectan con Chile (Del Carmen Parma, 1994).

7 Los Planes Jefes y Jefas de Hogar son parte de un programa nacional para desempleados con familias a cargo, se les entrega una remuneración mensual de $\$ 150$ (equivalente a USD 50) y a cambio ellos deben contrartestar realizando algún tipo de trabajo para la comunidad en instituciones estatales y ONGs. 
La superficie del departamento de Las Heras es de 10.035 kilómetros, lo que representa el $6,7 \%$ de la superficie provincial. A pesar de ser un departamento con mucho territorio, su relevancia productiva es ínfima debido a que gran parte es ocupado por la Cordillera de los Andes.

La población del departamento de Las Heras es de 182.962 personas $^{8}$, lo que equivale a un $11,6 \%$ de la población de la provincia de Mendoza' ${ }^{\text {. La }}$ población del distrito El Pastal es de 1.339 habitantes, según el último Censo Nacional de Población. Si se analiza a qué porcentaje de la población total del departamento equivale, se observa que es sólo el $0,73 \%$ de la población departamental; aun así es relevante el análisis que se desprende del estudio de la zona, porque este distrito rural se ubica a sólo 15 kilómetros de la ciudad de Las Heras, y es un distrito rural muy pobre.

Se puede observar que las grandes carencias de la población rural, en cuanto al acceso a algunos servicios básicos, como salud, saneamiento ambiental, educación, no son cubiertas de manera automática como apoyo a sus actividades productivas.

Las calles del distrito son de tierra y no hay ninguna asfaltada. Vinculado con esto, los informantes comentaron que la frecuencia de los colectivos es muy escasa, lo cual dificulta la salida y el regreso al distrito. A pesar de la cercanía de la ciudad, el distrito no tiene acceso a servicios básicos como gas natural, cloaca, transporte de calidad, y en algunos casos electricidad y agua potable. No existe ninguna industria en funcionamiento en la zona.

El distrito posee un centro de salud, que se encuentra en buen estado, pero tiene un número insuficiente de médicos, sobre todo de médicos especialistas. Los informantes también comentaron que faltan remedios. Se cuenta con dos escuelas primarias (EGB) y ninguna escuela secundaria (Polimodal).

De la medición del nivel educativo de los encuestados, se puede concluir que la mayoría de la población ( $80 \%$ aproximadamente) tiene, en el mejor de los casos, la EGB completa.

Las políticas de desarrollo rural necesitan ser acompañadas de un efectivo proceso de apoyo al desarrollo y fortalecimiento de las organizaciones de la población, que contribuya a sus posibilidades de transformarse efectivamente en los actores centrales del proceso. Para ello, se indagaron aspectos vinculados a la participación, de modo tal que el diagnóstico permitiera

Segun el INDEC, Censo Nacional de Población, Hogares y Vivienda 2001.

9 Según el INDEC, Censo Nacional de Población, Hogares y Vivienda 2001, el total de la población de la provincia de Mendoza es de 1.579.65! habitantes. 
conocer el grado de participación de los productores. Cuando se analizó la temática de la participación, se pudo observar que la participación de los habitantes no existe en instituciones no gubernamentales.

El municipio utilizó dicho informe para lucirse discursivamente frente a otros municipios e instituciones gubemamentales provinciales, pero no hizo nada concreto para revertir las situaciones de extrema pobreza y bajos niveles educativos de los pobladores de la zona rural analizada.

Como puede observarse, la descentralización de la educación y de la mayoría de los servicios públicos fue un proceso en el cual se descentralizaron las funciones, no así los recursos. Históricamente, los municipios sólo desarrollaban su función de barrido, alumbrado y limpieza, y se reconocían más como institución política que como un proveedor de bienes y servicios. El Estado Nacional ha transferido nuevas competencias a niveles inferiores sin transferir los recursos necesarios; por otra parte, la sociedad civil pide mayor eficiencia y control de la gestión municipal y exige espacios de participación activa. La crisis, la desocupación y la tensión social también convierten al municipio como punto de condensación de la fragmentación social.

Las administraciones locales en la actualidad tienen nuevas competencias y actividades: administrar eficientemente los recursos físicos, humanos, financieros y económicos, trabajar en pro de la disminución/erradicación de la pobreza, preservar el equilibrio ecológico y diseñar políticas de empleo, promover la planificación del desarrollo económico y social, atender la problemática de las actividades productivas (PYMES y microempresas), etc.

El municipio tiene que enfrentar el problema de la autonomía que abarca varias esferas: financiera, institucional y administrativa, pero no cuenta con el personal capacitado ni con la voluntad política para generar cambios educativos y de accesibilidad para estos segmentos de la población, que no sólo son rurales sino además marginales, improductivos, y de poca importancia por la cantidad de votos.

\section{Consideraciones finales}

A comienzos del siglo XXI, la escuela tiene que asumir nuevas funciones y responsabilidades, no vinculadas directamente al proceso de enseñanza aprendizaje, sino como articuladora con la comunidad. En el caso de la escuela rural, este rol cobra mayor relevancia, ya que la escuela es visualizada como la institución pública con mayor presencia en el territorio. Los roles que debe asumir permiten que se posicione como agente de desarrollo local 
y ente capaz de reafirmar las identidades a través de reforzar los procesos de socialización de los estudiantes y de su entorno inmediato. Sin embargo, debe recordarse que la escuela no puede imponer modelos culturales a la comunidad, sino que tiene que aprender a construir, en conjunto, desde la cultura, ideología y necesidades de la gente. Es así que la educación como proceso de socialización que dura toda la vida, permite a la escuela generar y acompañar procesos de empoderamiento de la comunidad.

En el contexto neoliberal que atraviesa el mundo por estos días, debe exigirse a los gobiemos que generen procesos de descentralización que permitan a las comunidades apropiarse de herramientas como la educación, pero con el cuidado de que no sólo se descentralicen las funciones, sino también los recursos y se capacite a quienes deben hacerse cargo de estas en los niveles locales; de otro modo, la educación, sobre todo en las zonas rurales de América Latina, seguirá siendo excluyente y desigual en sus prácticas.

Por último, se debe poner en consideración el rol de la historia en los procesos sociales, como elemento dinamizador que permite la acumulación de conocimientos y experiencias para que pueblos como los latinoamericanos, no sigan permitiendo el avasallamiento cultural y político que los países desarrollados pretenden imponer e importar a las comunidades en nombre del desarrollo. 


\section{REFERENCIAS}

Arocena, J. (1995). "El desarrollo rural como desafio teórico", en El desarrollo local: Un desafio contemporáneo. Centro Latinoamericano de Economía Humana (CLAEH), Universidad Católica de Uruguay. Unuguay: Editorial Nueva Sociedad.

Arocena, J. (1998). "Discutiendo la dimensión de lo local. Las coordenadas del debate", en Descentralización y desarrollo local. Análisis, experiencias y propuestas. Centro Latinoamericano de Economía Humana (CLAEH), Universidad Católica de Uruguay. Uruguay: Editorial Nueva Sociedad.

Bahamondes, M. La producción campesina (Aspectos sociales, culturales y económicos). Santiago, Chile. Documento de trabajo $\mathrm{N}^{\circ} 10$, Universidad Academia de Humanismo Cristiano. Santiago, Chile: Grupo de Investigaciones Agrarias.

Barsky, O. y Gelman, J. (2001). Análisis de la historia del agro argentino. Buenos Aires, Argentina: Grupo Editorial Grijalvo-Mondadori.

Bocco, A.; Martín, C.; Panuzzio, M. (1999). Mendoza, agricultura y ruralidad. Reflejos en la estructura social y agraria del oasis rural de San Carlos. Argentina: EDIUNC.

Calva, J. L. (1988). Los campesinos y su devenir en las sociedades de mercado. México: Ediciones Siglo XXI.

Canafoglia, E.; Millán, N.; Soria, B. (2006). "La contracara de los indicadores del mercado de trabajo en la Provincia de Mendoza". Laboratorio/n Line, Año VII, Número 19, otoño / inviemo 2006. Instituto de Investigaciones Gino Germani, Facultad de Ciencias Sociales, Universidad de Buenos Aires, Argentina.

Coraggio, J. L. Del sector informal a la economia popular: un paso estratégico para el planteamiento de alternativas populares de desarrollo social. (FLACSOEcuador/ www.FRONESIS).

Craviotti, C. y Soverna, S. (1999). La sistematización de estudios de casos de pobreza rural. Serie Documentos de Formulación, Dirección de Desarrollo Agropecuario, PROINDER, Secretaría de Agricultura, Ganadería, Pesca y Alimentación de la Nación, Buenos Aires, Argentina.

Cullen, C. (1996). Autonomia moral, participación democrática y cuidado del otro. Bases para un curriculum de formación ética y ciudadana. Buenos Aires, Argentina: Ediciones Novedades Educativas

Cullen, C. (1997). Critica de las razones de educa. Buenos Aires, Argentina: Editorial Paidós.

Del Carmen Parma, N. (1994). El departamento de Las Heras, estandarte libertador: Argentina: Ediciones Culturales de Mendoza, Mendoza. 
Di Pietro, P. (2003). "Hacia un desarrollo integrador y equitativo: una introducción al desarrollo local", en Burin, D. y Heras, A. (comp.). Desarrollo local una respuesta a escala humana a la globalización. Ediciones CICCUS - La Crujía.

Díaz, E. (editora). (1996). La ciencia y el imaginario social. Buenos Aires, Argentina: Editorial Biblos.

Dussel, I. (1996). La escuela y la formación de la ciudadania: reflexiones en tiempos de crisis. FLACSO Sede Argentina, Área Educación y Sociedad, Serie de Documentos e Informes de Investigación $N^{\circ} 186$, Buenos Aires, Argentina.

Furche, C. (1990). La economia campesina y su inserción estructural: elementos para una discusión. Serie Materiales para Capacitación, Curso de Capacitación a Profesionales y Técnicos de Organizaciones no Gubernamentales de Apoyo al Sector Rural No 1, GIA, Santiago, Chile.

Gentili, P. (coordinador). (2000). Códigos para la ciudadania. La formación ética como parte de la libertad, saberes clave para educadores. Buenos Aires, Argentina: Editorial Santillana.

Gilly, J. P. y Torres, A. (2000). "Capítulo 8: Proximidad y dinámicas territoriales", en Territorio, conocimiento y competitividad de las empresas (compiladores Boscherini, F. y Poma, L.). Buenos Aires, Argentina: Miño y Dávila Editores.

Giroux, H. (1993). La escuela y la lucha por la ciudadania (1 era. edición en español). México: Siglo Veintiuno Editores.

Gómez, S. (2002). Nueva ruralidad ¿Qué tan nueva? Edición Universidad Austral de Chile, Facultad de Filosofia y Humanidades.

González, L. (1998). "Educación, valores y democracia". Sinéctica (12).

INDEC. (2001). Censo Nacional de Población, Hogares y Vivienda.

INDEC. (2002). Censo Nacional Agropecuario.

Landi, O. (2002). "La metamorfosis del ciudadano", Diario Clarin, Suplemento Zona, domingo 24 de marzo de 2002.

Manzanal, M. (2005). Regiones, territorios e institucionalidad del desarrollo rural, Primeras jornadas de intercambio y discusión: El desarrollo rural en su perspectiva institucional y territorial. Organizado por la Universidad Nacional de Buenos Aires, la Universidad Nacional de Rosario y el CEIL-PIETTE del CONICET, Buenos Aires, Argentina.

Millán, N. (2006). Pobreza rural en Mendoza. Instituciones, desarrollos teóricos y metodologias aplicadas para su conocimiento y eliminación. Informe de pasantía SIEMPRO-Universidad Nacional de Cuyo.

Ministerio de Ciencia y Tecnología, Presidencia de la Nación. Educación. Plan de Acción 2005-2007.

Ministerio de Cultura y Educación de la Nación. (1994). Conozcamos la Ley. Buenos Aires, Argentina. 
Ministerio de Cultura y Educación de la Nación. (1997). "Cuestión de enfoques". Zona educativa 2 (14), Argentina.

Osorio Vargas, J. (Primer semestre de 1995). "Ciudadanía y posibilidades de una educación del sentido de lo común". La Piragua, Revista Latinoamericana de Educación y Política (10), Santiago de Chile.

Osorio Vargas, J. (Primer semestre de 1994). "La educación como formación de sujetos y la construcción de la ciudadanía en América Latina". La Piragua (8), Santiago de Chile.

Puiggrós, A. (1990). Sujetos, disciplina y curriculum en los origenes del sistema educativo argentino. Buenos Aires, Argentina: Editorial Galerna.

Raiter, A. (compilador). (2002). Representaciones sociales. Buenos Aires, Argentina: Editorial Eudeba.

Ríos, S. (2000). Los agrupamientos de población en el territorio. Apuntes Docentes. Programa de Capacitación y Extensión Rural. GIA, Santiago, Chile.

Sautu, R. (2003). Todo es teoría. Buenos Aires, Argentina: Ediciones Lumiere.

Schejtman, A. y Berdegué, J. (2003). Desarrollo territorial rural. Santiago, Chile: RIMISP.

Torres, C. A. (1994). Estado, privatizaciones y política educativa. Elementos para una crítica del neoliberalismo. Ponencia presentada en el Coloquio Internacional sobre relaciones entre gobierno, justicia y cultura. Organizado por la Embajada de Francia y el Instituto de Investigaciones Sociales, Universidad Autónoma de México, en la ciudad de México, 4-6 de octubre, 1994, compilado en el libro Las secretas aventuras del orden, el Estado y la Educación, Miño y Dávila Editores.

Torres, C. A. y González Rivera, G. (1994). Sociologia de la educación, corrientes contemporáneas. Buenos Aires, Argentina: Miño y Dávila Editores. 